\title{
FINITE TERM RELATIONS FOR THE EXPONENTIAL ORTHOGONAL POLYNOMIALS*
}

\author{
Björn Gustafsson ${ }^{1}$ And Mihai Putinar Pun,** $^{2,3, *}$
}

\begin{abstract}
The exponential orthogonal polynomials encode via the theory of hyponormal operators a shade function $g$ supported by a bounded planar shape. We prove under natural regularity assumptions that these complex polynomials satisfy a three term relation if and only if the underlying shape is an ellipse carrying uniform black on white. More generally, we show that a finite term relation among these orthogonal polynomials holds if and only if the first row in the associated Hessenberg matrix has finite support. This rigidity phenomenon is in sharp contrast with the theory of classical complex orthogonal polynomials. On function theory side, we offer an effective way based on the Cauchy transforms of $g, \bar{z} g, \ldots, \bar{z}^{d} g$, to decide whether a $(d+2)$-term relation among the exponential orthogonal polynomials exists; in that case we indicate how the shade function $g$ can be reconstructed from a resulting polynomial of degree $d$ and the Cauchy transform of $g$. A discussion of the relevance of the main concepts in Hele-Shaw dynamics completes the article.
\end{abstract}

Mathematics Subject Classification. 33C47, 32A26, 47B20, 47B35.

Received February 3, 2019. Accepted May 14, 2019.

\section{INTRODUCTION}

In contrast to the unanimously accepted conventions in the theory of orthogonal polynomials on the line or on the circle, the multivariate setting is asking for additional, rather essential choices, such as working with complex variables rather than real ones, ordering the monomials, smoothness properties of the generating measures, (re)normalizations in the asymptotic analysis. It is well known for instance that the finite central truncation approach (of Jacobi matrices) to orthogonal polynomials encounters serious complications when passing to several variables, see for instance [5]. These difficulties are mostly related to the non-commutativity of the finite rank compressions of commuting, infinite (Jacobi type) matrices.

A rather specialized setting, relevant for instance for image processing, is offered by the theory of hyponormal operators. More specifically, a spectral parameter known as the principal function of a hyponormal operator, turns the moments of a "shade function" depending of two real variables into a meaningful and accessible Hilbert space operator. The associated complex orthogonal polynomials, called in [9] and henceforth the exponential

${ }^{*}$ We are grateful to the referees for their constructive observations which improved the accessibility of this article.

Keywords and phrases: Complex orthogonal polynomials, exponential transform, finite term relation, hyponormal operator, quadrature domain.

${ }^{1}$ Department of Mathematics, KTH, 10044 Stockholm, Sweden.

2 Department of Mathematics, University of California, Santa Barbara, CA 93106-3080, USA.

3 School of Mathematics, Statistics and Physics, Newcastle University, Newcastle upon Tyne, NE1 7RU, UK.

** Corresponding author: mputinar@math.ucsb.edu 
orthogonal polynomials, reveal in algebraic terms the geometry of the generating shaded shape. For instance, the analogs of finite collections of intervals on the line or on the circle are quadrature domains for complex analytic functions in the plane. The analogy to the one dimensional classical theory, and especially to Markov's exponential transform of the generating function of a moment sequence, goes quite far. The recent lecture notes [9] contain a detailed account of the qualitative features of exponential orthogonal polynomials and the necessary Hilbert space or potential theory background.

The present note is aimed at proving a notable rigidity of the Hessenberg matrix associated to a system of exponential orthogonal polynomials. Namely, assuming a necessary completeness of the exponential orthogonal polynomials, a finite number of non-zero entries on the first row propagates to the whole matrix and produces a finite banded matrix with one non-zero sub-diagonal. Finite banded matrices have been intensively studied for a long time, both for theoretical features as well as for their relevance in numerical linear algebra, see for instance [4].

Moreover, if only the first two elements of the first row of Hessenberg's matrix are non-zero and the exponential orthogonal polynomials are complete, then the whole matrix is tridiagonal and the associated shaded shape is a an ellipse (black on white). This observation complements existing similar results for classical complex orthogonal polynomials $[6,14,17,20]$.

In the finite term relation scenario, the exponential transform of the generating function of the power moments of a shade function $g$ satisfies a characteristic vanishing formula. More specifically, the Taylor coefficients at infinity of this transform reveal elementary recursion relations (at most quadratic) which populate the entire double series from its marginals. As a byproduct, we indicate an algorithmic approach to determine from the Cauchy transforms of $g, \bar{z} g, \ldots, \bar{z}^{d} g$ the existence of a finite term relation for the associated exponential orthogonal polynomials, and if so, how to reconstruct $g$. The exact reconstruction of a domain from exponential data transform goes back a couple of decencies, see [9] for a recent account of the algorithm and some updated comments.

While the main body of the present work is presented from a stationary point of view, a natural question raised in the last section addresses the evolution of shade functions (possibly carrying a finite term relation) under Hele-Shaw flow. The better understood case of families of confocal ellipses offers a promising base to start such a quest.

\section{Preliminaries}

The starting point of the dictionary between shade functions in the plane and Hilbert space operators is the following double Cauchy transform positivity feature. Let $g: \mathbb{C} \longrightarrow[0,1]$ be a Borel measurable function with compact support, and denote by

$$
a_{j k}=\frac{1}{\pi} \int_{\mathbb{C}} z^{j} \bar{z}^{k} g(z) \mathrm{d} A, \quad j, k \geq 0,
$$

its moments. Above $\mathrm{d} A$ stands for Lebesgue measure in the plane. The double Cauchy transform

$$
-\frac{1}{\pi} \int_{\mathbb{C}} \frac{g(\zeta) \mathrm{d} A(\zeta)}{(\zeta-z)(\bar{\zeta}-\bar{w})}
$$

expands in the convergent generating series in a neighborhood of infinity:

$$
-\sum_{j, k=0}^{\infty} \frac{a_{j k}}{z^{j+1} \bar{w}^{k+1}}
$$


The exponential of this series, called the "exponential transform" of the shade function $g$ has the remarkable positivity property of factoring through a Hilbert space $H$ and a linear bounded operator $T$ acting on $H$ :

$$
E_{g}(z, w)=\exp \left[-\frac{1}{\pi} \int_{\mathbb{C}} \frac{g(\zeta) \mathrm{d} A(\zeta)}{(\zeta-z)(\bar{\zeta}-\bar{w})}\right]=1-\left\langle\left(T^{*}-\bar{w}\right)^{-1} \xi,\left(T^{*}-\bar{z}\right)^{-1} \xi\right\rangle .
$$

The latter identity extends from a neighborhood of infinity to the whole $\mathbb{C}^{2}$, with proper definitions of the localized resolvents. In this factorization the operator $T$ is almost normal, in the precise sense that its selfcommutator is small, but not zero:

$$
\left[T^{*}, T\right]=\xi\langle\cdot, \xi\rangle=: \xi \otimes \xi
$$

And vice-versa, an irreducible operator with non-negative self-commutator of rank one produces by the above exponential transform a shade function $g$, called the principal function. For all aspects of the theory of hyponormal operators invoked in this article we refer to [9] and the bibliographical references therein.

The exponential orthogonal polynomials are trimmed to the Hilbert space realization rather than the function theoretic setting. Specifically, $P_{k}(z)$ is the unique polynomial of degree $k$ with positive leading coefficient which satisfies the state space orthogonality

$$
\left\langle P_{k}\left(T^{*}\right) \xi, T^{* j} \xi\right\rangle=0, \quad j<k,
$$

and the normalization

$$
\left\|P_{k}\left(T^{*}\right) \xi\right\|=1, \quad k \geq 0
$$

This Krylov subspace type scheme goes through to any integer $k$ if and only if the filtration of cyclic subspaces

$$
H_{k}=\operatorname{span}\left\{\xi, T^{*} \xi, \ldots, T^{* k} \xi\right\}, k \geq 0,
$$

is not stationary.

The stationary case is interesting, and was studied in full detail before, being represented by black and white shade functions $g=\chi_{\Omega}$, where $\Omega$ is a quadrature domain for analytic functions in the complex plane, [9].

All these being said, one can avoid the rather abstract operator $T$ and define a multiplier on a functional model space involving only Cauchy transforms and multiplier operations, see [9] or the recent note [10].

\section{Completeness of exponential orthogonal polynomials}

We use the notation introduced in Section 2: $T \in L(H)$ is a pure hyponormal operator with rank-one selfcommutator, $P_{k}(z)$ are the corresponding exponential orthogonal polynomials. In addition we let $\mathcal{H}$ represent the Hessenberg matrix associated to the multiplier $M_{z}$ with respect to the orthonormal system $P_{k}(z)$. Note that $P_{k}$ is defined without ambiguity if and only if the space $H_{k-1}$ (see (2.2)) is strictly contained in $H_{k}$. If this is not the case, that is $H_{k-1}=H_{k}$, then the spectrum of the operator $T$ is the closure of a quadrature domain (or open set) $\Omega$ of order less than or equal to $k$, carrying a "maximal" principal function $g_{T}=\chi_{\Omega} \mathrm{d} A$; moreover in this case $P_{k}$ makes no sense. We will carry this dichotomy, namely either $H_{k-1} \neq H_{k}$ for all $k \geq 1$, or there exists $d \geq 1$, minimal with the property $H_{d-1}=H_{d}$. In either situation we will speak about the exponential orthogonal polynomials $P_{k}$, defined only for all degrees $k \geq 0$, respectively only for $0 \leq k \leq d-1$. Correspondingly, the Hessenberg matrix $\mathcal{H}$ will be infinite or finite. More specifically, $\mathcal{H}=\left(h_{j k}\right)$, where

$$
h_{j k}=\left\langle z P_{k}, P_{j}\right\rangle
$$


with $j, k \geq 0$, respectively $0 \leq j, k \leq d-1$. Note that the matrix $\mathcal{H}$ has only a first non-zero subdiagonal:

$$
\mathcal{H}=\left(\begin{array}{cccccc}
h_{00} & h_{01} & h_{02} & h_{03} & h_{04} & \ldots \\
h_{10} & h_{11} & h_{12} & h_{13} & h_{14} & \ldots \\
0 & h_{21} & h_{22} & h_{23} & h_{24} & \ldots \\
0 & 0 & h_{32} & h_{33} & h_{34} & \ldots \\
\vdots & & \vdots & & \ddots & \ddots
\end{array}\right)
$$

Let $H_{\text {pol }}$ denote the closed linear subspace of $H$ generated by the orthonormal system $e_{k}=P_{k}\left(T^{*}\right) \xi, k \geq 0$ :

$$
H_{\mathrm{pol}}=\bigvee_{k \geq 0} P_{k}\left(T^{*}\right) \xi=\bigvee_{k \geq 0} T^{* k} \xi
$$

We may call $H_{\text {pol }}$ the closure of exponential polynomials in the underlying Hilbert space $H$. This subspace might be smaller than $H$, even finite dimensional, yet it carries full information on the operator $T$, and hence on the associated shade function $g_{T}$. Indeed, the machinery of the exponential transform carries bijectively the Gram matrix data

$$
b_{\ell, k}=\left\langle T^{* k} \xi, T^{* \ell} \xi\right\rangle, \quad k, \ell \geq 0,
$$

to the $(z, \bar{z})$-moments of $g_{T}$. One step further, standard linear algebra (LU factorization of the inverse Gram matrix) produces from $\left(b_{\ell, k}\right)$ the coefficients of the orthogonal polynomials $P_{k}$ (for all degrees they are well defined) and finally the "shift" matrix $\mathcal{H}$ together with the length of the vector $\xi=\|\xi\| e_{0}$ determine the Gram matrix, and hence $T$. We do not expand here these known details, see for instance [3].

The sub-diagonal in the Hessenberg matrix is of particular interest, at least in the general theory of orthogonal polynomials. We confine ourselves to note the simple dependence of its entries and the leading coefficients of the orthogonal polynomials. Specifically, if

$$
P_{n}(z)=\gamma_{n} z^{n}+\cdots
$$

where $\gamma_{n}>0, n \geq 0$, then

$$
h_{n+1, n}=\left\langle z P_{n}(z), P_{n+1}(z)\right\rangle=\left\langle\gamma_{n} z^{n+1}+\cdots, P_{n+1}(z)\right\rangle=\frac{\gamma_{n}}{\gamma_{n+1}} .
$$

Recall also Christoffel function type interpretation

$$
\frac{1}{\gamma_{n}}=\operatorname{dist}\left(z^{n}, H_{n-1}\right)
$$

which is the starting point of asymptotic formulas in the classical situation.

As a conclusion of this general discussion, we emphasize the following rather striking phenomenon specific to our framework. Let $\pi: H \longrightarrow H_{\text {pol }}$ denote the orthogonal projection. The operator $T^{*}$ leaves invariant the subspace $H_{\mathrm{pol}}$, hence $T^{*} \pi=\pi T^{*} \pi=\mathcal{H}$. In matrix form, we obtain the block decomposition:

$$
T=\left(\begin{array}{cc}
\mathcal{H}^{*} & 0 \\
B & C
\end{array}\right)
$$

The upper-left corner $\mathcal{H}^{*}$ determines the whole operator $T$ in a rather constructive manner (see the stair-case block-matrix structure discussed in [9] for the case of quadrature domains, that is $\left.\operatorname{dim} H_{\mathrm{pol}}<\infty\right)$. In general 
the recursive procedure to compute all moments

$$
\left\langle T^{p} T^{* q} \xi, T^{k} T^{* \ell} \xi\right\rangle, \quad p, q, \ell, k \geq 0,
$$

from the compressed data

$$
b_{k, \ell}=\left\langle T^{* \ell} \xi, T^{* k} \xi\right\rangle, \quad k, \ell \geq 0
$$

is derivable from the basic commutation relation $T^{*} T=T T^{*}+\xi \otimes \xi$. See for details Section XII.3 in [15].

From these observations we derive a simple quantitative criterion for the completeness of exponential orthogonal polynomials, that is for the equality $H_{\mathrm{pol}}=H$ to hold.

Theorem 3.1. Let $T \in L(H)$ be an irreducible hyponormal operator with rank-one self-commutator and associated shade function $g$. Let $P_{k}(z)$ denote the associated exponential orthogonal polynomials and let $\mathcal{H}=\left(h_{j k}\right)$ be the associated Hessenberg matrix (finite or not).

The system of exponential orthogonal polynomials is complete if and only if one of the following conditions is satisfied:

$$
\sum_{k \geq 1}\left|h_{0 k}\right|^{2}-\left|h_{10}\right|^{2}=\frac{\|g\|_{1}}{\pi},
$$

or equivalently

$$
T \xi \in H_{\mathrm{pol}} .
$$

Proof. We first claim that the closure of polynomials $H_{\mathrm{pol}}=\bigvee_{k>0} T^{* k} \xi$ is equal to the full space $H$ if and only if (3.2) holds. Indeed, assuming $T \xi \in H_{\text {pol }}$, we infer by induction from the commutation relation $T T^{*}=T^{*} T-\xi \otimes \xi$ that $T T^{* k} \xi \in H_{\text {pol }}$ for all $k \geq 0$ (see Eq. (4.3) below for some details). But this means that $H_{\text {pol }}$ is a reducing subspace for the irreducible operator $T$, that is $T H_{\text {pol }} \subset H_{\text {pol }}$ and $T^{*} H_{\text {pol }} \subset H_{\text {pol }}$, hence $H_{\text {pol }}=H$.

Next, $T \xi \in H_{\text {pol }}$ if and only if the orthogonal projection $\pi$ onto $H_{\text {pol }}$ satisfies

$$
\pi T \xi=T \xi
$$

or equivalently

$$
\|T \xi\|=\|\pi T \xi\|=\left\|\mathcal{H}^{*} \xi\right\| .
$$

Let $e_{0}=\xi /\|\xi\|$ be the first orthonormal vector in the system associated to the exponential orthogonal polynomials. The last equality becomes:

$$
\left\|T e_{0}\right\|^{2}=\left\|\mathcal{H}^{*} e_{0}\right\|^{2}=\sum_{k \geq 0}\left|h_{0 k}\right|^{2}
$$

On the other hand the commutation relation $T^{*} T=T T^{*}+\xi \otimes \xi$ implies

$$
\left\|T e_{0}\right\|^{2}=\left\|T^{*} e_{0}\right\|^{2}+\left|\left\langle e_{0}, \xi\right\rangle\right|^{2}=\left|h_{00}\right|^{2}+\left|h_{10}\right|^{2}+\|\xi\|^{2} .
$$


Finally, the very definition of the exponential transform and its factorization through the resolvent of the operator $T^{*}$ yield:

$$
\|\xi\|^{2}=\frac{1}{\pi} \int_{\mathbb{C}} g \mathrm{~d} A
$$

By putting together these computations we obtain the equivalence in the statement.

Corollary 3.2. The first row and first column in the Hessenberg matrix attached to any irreducible hyponormal operator with rank-one self-commutator and associated shade function g satisfy the inequality:

$$
\sum_{k \geq 1}\left|h_{0 k}\right|^{2}-\left|h_{10}\right|^{2} \leq \frac{\|g\|_{1}}{\pi} .
$$

For the proof it suffices to note that

$$
\|\pi T \xi\| \leq\|T \xi\|
$$

Besides the analytic criterion offered by the theorem above, we can derive in purely geometric terms sufficient conditions for the completeness of exponential orthogonal polynomials.

Proposition 3.3. Let $g=\chi_{\sigma}$ be a black and white shade function of compact support $\sigma$. The associated exponential orthogonal polynomials are complete if the complement of $\sigma$ is connected and either int $\sigma=\emptyset$ or $\partial \sigma$ is real analytic smooth and int $\sigma$ is not a quadrature domain.

Proof. We exploit the weak continuity of the generalized resolvent $\left(T^{*}-\bar{z}\right)^{-1} \xi$, defined over the whole complex plane, [9]. First we eliminate the case that int $\sigma$ is a quadrature domain, characterized by the finite dimensionality of the space $H_{\mathrm{pol}}$.

Assume $H_{\mathrm{pol}} \neq H$, that is there exists a non-zero vector $u \in H \ominus H_{\mathrm{pol}}$. Consider the continuous function

$$
\alpha(z)=\left\langle u,\left(T^{*}-\bar{z}\right)^{-1} \xi\right\rangle, \quad z \in \mathbb{C} .
$$

The analyticity of $\alpha(z)$ in the complement of $\sigma$ and a Neumann series at infinity imply

$$
\alpha(z)=0, \quad z \in \mathbb{C} \backslash \sigma
$$

In case int $\sigma=\emptyset$, we infer from continuity that $\alpha$ is identically zero. On the other hand, the span of the vectors $\left(T^{*}-\bar{z}\right)^{-1} \xi, z \in \mathbb{C}$, is dense in $H$ (see again [9]), hence $u=0$, a contradiction.

In case $\Omega:=\operatorname{int} \sigma$ is not empty and $\Gamma=\partial \sigma$ is real analytic smooth we argue as follows. The function $\alpha(z)$ defined above vanishes on the complement of $\sigma$ and it is bianalytic in int $\sigma$. In general $\alpha$ is bianalytic (i.e., $\left.\partial^{2} \alpha / \partial \bar{z}^{2}=0\right)$ in regions where $g^{2}=g$, as can be seen by examining the exponential transform. That is

$$
\alpha(z)=\bar{z} h_{1}(z)+h_{2}(z), \quad z \in \Omega
$$

with $h_{1}, h_{2}$ analytic functions in $\Omega$. In view of (3.3):

$$
\alpha=0 \text { on } \Gamma \text {. }
$$

If $h_{1}$ is identically zero, then so is $h_{2}$ by the maximum principle and there is nothing left to prove. From now on we assume that $h_{1}$ is not identically zero. 
The analyticity of $\Gamma$ also implies the estimate

$$
\int_{\Omega}\left\|\frac{\partial}{\partial \bar{z}}\left(T^{*}-\bar{z}\right)^{-1} \xi\right\| \mathrm{d} A(z)<\infty .
$$

For a proof, see for instance Theorem 5.6 in [8]. As a consequence of this bound one finds

$$
h_{1}=\frac{\partial \alpha}{\partial \bar{z}} \in L^{1}(\Omega, \mathrm{d} A),
$$

hence the function $h_{2}$ is also summable in $\Omega$.

A second implication of the analyticity of the boundary $\Gamma$ is the existence of a compact subset $K \subset \Omega$ and a positive constant $C$, so that

$$
\left|\int_{\Omega} f \mathrm{~d} A\right| \leq C\|f\|_{\infty, K}, \quad f \in \mathcal{O}(\bar{\Omega})
$$

that is for every analytic function $f$ defined in a neighborhood of $\sigma=\bar{\Omega}$. Indeed, due to the smooth analyticity of $\Gamma$ there exists an analytic function $S(z)$ defined in a neighborhood of $\Gamma$, subject to the constraint

$$
S(\zeta)=\bar{\zeta}, \quad \zeta \in \Gamma
$$

This is the so called "Schwarz function" of the curve $\Gamma[1,19]$. Under these assumptions Stokes' theorem yields

$$
\int_{\Omega} f \mathrm{~d} A=\frac{1}{2 i} \int_{\Omega} f(z) \mathrm{d} \bar{z} \wedge \mathrm{d} z=\frac{1}{2 i} \int_{\Gamma} f(\zeta) S(\zeta) \mathrm{d} \zeta
$$

and the latter line integral can be pushed inside $\Omega$ by Cauchy's theorem and the analyticity of $S$.

Let $\left(\Omega_{n}\right)$ be an increasing exhaustion of $\Omega$ with relatively compact domains with smooth boundaries. For a function $f \in \mathcal{O}(\bar{\Omega})$ we find

$$
\begin{aligned}
\int_{\Omega} h_{1} f \mathrm{~d} A & =\lim _{n} \int_{\Omega_{n}} h_{1} f \mathrm{~d} A=\lim _{n} \frac{1}{2 i} \int_{\partial \Omega_{n}} \bar{z} h_{1} f \mathrm{~d} z \\
& =\lim _{n} \frac{1}{2 i} \int_{\partial \Omega_{n}}\left(\bar{z} h_{1}+h_{2}\right) f \mathrm{~d} z=\frac{1}{2 i} \int_{\Gamma} \alpha f \mathrm{~d} z=0 .
\end{aligned}
$$

Since the analytic function $h_{1}$ admits only finitely many zeros on $K$, there exists a polynomial $P(z)$ vanishing exactly at these zeros, counting also multiplicities. We deduce

$$
\int_{\Omega} \operatorname{Pfd} A=0, \quad f \in \mathcal{O}(\bar{\Omega})
$$

Lagrange interpolation formula proves then that $\Omega$ is a quadrature domain.

We will see shortly that one cannot avoid in the statement of the proposition the assumption on $\mathbb{C} \backslash \sigma$ to be connected. A challenging question is to derive similar sufficient conditions for the equality $H_{\text {pol }}=H$ in the presence of a true shade function $g\left(\right.$ i.e. $g^{2} \neq g$ ). One step further, one can refine the above criterion to a non-simply connected domain, by replacing the polynomial closure $H_{\text {pol }}$ by the larger space

$$
H_{\mathrm{an}}=\operatorname{span}_{\lambda \notin \sigma(T)}\left(T^{*}-\bar{\lambda}\right)^{-1} \xi
$$


This generalization steps outside the realm of orthogonal polynomials and we do not pursue it here.

\section{THREE TERM RELATION}

In this section we prove among other things that ellipses are the only black and white shapes in the complex plane (with connected component and without slits) which carry a three term relation in the corresponding quantization by exponential orthogonal polynomials.

Let $\left(P_{n}(z)\right)_{n=0}^{\infty}$ be the exponential orthogonal polynomials associated to a compactly supported shade function $g: \mathbb{C} \longrightarrow[0,1]$ which is not the characteristic function of a quadrature domain. The Hessenberg matrix $\mathcal{H}=\left(h_{j k}\right)$, is therefore infinite:

$$
h_{j k}=\left\langle z P_{k}, P_{j}\right\rangle, \quad j, k \geq 0,
$$

and has only the first sub-diagonal non-zero. If only the first upper-diagonal is non-zero one encounters a classical three term relation

$$
z P_{k}(z)=h_{k+1, k} P_{k+1}(z)+h_{k k} P_{k}(z)+h_{k-1, k} P_{k-1}(z), \quad k \geq 0,
$$

where $P_{-1}=0$ by convention. It is well known that orthogonal polynomial in the Lebesgue space $L^{2}$ of a positive measure supported by the real line satisfy a three term relation. A necessary cosmetic modification also produces a three term relation for orthogonal polynomials on the circle.

Our main result reduces the three term relation to a much weaker recursion identity, identified by the first row of Hessenberg's matrix. Namely

$$
z P_{k}(z)=\sum_{j=1}^{k+1} h_{j k} P_{j}(z), \quad k \geq 2,
$$

instead of having the summation starting with $j=0$. In other terms

$$
\left\langle z P_{k}(z), P_{0}(z)\right\rangle=0, k \geq 2,
$$

or equivalently, Hessenberg matrix $\mathcal{H}$ has only the first two terms possibly non-zero on the first row:

$$
\mathcal{H}=\left(\begin{array}{cccccc}
h_{00} & h_{01} & 0 & 0 & 0 & \ldots \\
h_{10} & h_{11} & h_{12} & h_{13} & h_{14} & \ldots \\
0 & h_{21} & h_{22} & h_{23} & h_{24} & \ldots \\
0 & 0 & h_{32} & h_{33} & h_{34} & \ldots \\
\vdots & & \vdots & & \ddots & \ddots
\end{array}\right)
$$

Recall that the first row of Hessenberg's matrix can be computed in the spirit of classical orthogonal polynomials by the formula:

$$
\left\langle z P_{k}(z), P_{0}(z)\right\rangle=\gamma \int_{\mathbb{C}} z P_{k}(z) g(z) \mathrm{d} A(z)
$$

where $\gamma$ is a constant, $[9](\gamma=1 /(\pi\|\xi\|)$, necessarily).

The precise statement is:

Theorem 4.1. Assume the exponential orthogonal polynomials $\left(P_{n}(z)\right)_{n=0}^{\infty}$ associated to a compactly supported shade function $g: \mathbb{C} \longrightarrow[0,1]$ are complete in the Hilbert space realization of the exponential transform of $g$, i.e. 
$H_{\mathrm{pol}}=H$. If, in addition, the recurrence relation among $P_{k}(z)^{\prime} s$ is missing the 0 -th order term for all $k \geq 2$, or equivalently if

$$
\int_{\mathbb{C}} z P_{k}(z) g(z) \mathrm{d} A(z)=0, \quad k \geq 2,
$$

then $g$ is the characteristic function of an ellipse.

Proof. Denote by $T \in L(H)$ the irreducible hyponormal operator $T$ with rank-one self-commutator $\left[T^{*}, T\right]=$ $\xi \otimes \xi$ and with principal function equal, a.e., to the prescribed shade function $g$. The assumption that the exponential orthogonal polynomials are complete is equivalent (by definition) to the fact that the filtration of finite dimensional spaces

$$
H_{k}=\operatorname{span}\left\{\xi, T^{*} \xi, \ldots, T^{* k} \xi\right\}, \quad k \geq 0,
$$

does not stop and it spans $H$. In other terms the system of orthonormal vectors $e_{k}=P_{k}\left(T^{*}\right) \xi, k \geq 0$, spans $H$.

Hessenberg's matrix $\left(h_{j k}\right)_{j, k=0}^{\infty}$ represents the linear transform $T^{*}$ in the basis $\left(e_{k}\right)$, while its conjugate transpose represents $T$. The assumption (4.1) and the completeness of orthogonal polynomials translate into

$$
T \xi \in H_{1},
$$

or equivalently

$$
T \xi=a \xi+b T^{*} \xi
$$

for some complex numbers $a, b$. Induction based on the identity

$$
T T^{* k}=T^{* k} T+\sum_{j=0}^{k-1} T^{* j}\left[T, T^{*}\right] T^{* k-j-1}=T^{* k} T-\sum_{j=0}^{k-1} T^{* j} \xi\left\langle\cdot, T^{k-j-1} \xi\right\rangle
$$

implies, together with (4.2), that

$$
T H_{k} \subset H_{k+1}, \quad k \geq 0 .
$$

In other terms, Hessenberg's matrix is tri-diagonal. According to Theorem 5.2 of [9], the shade function $g$ coincides then with the characteristic function of an ellipse.

We repeat for completeness the simple argument contained in [9]. Denote

$$
a_{k}=\overline{h_{k k}}, \quad b_{k+1}=\overline{h_{k, k+1}}, \quad c_{k+1}=\overline{h_{k+1, k}}, \quad k \geq 0,
$$

the three non-trivial diagonals in Hessenberg's matrix. The commutator assumption $\left[T^{*}, T\right]=\xi \otimes \xi$ reads on matrix elements:

$$
\begin{gathered}
\left|a_{0}\right|^{2}+\left|b_{1}\right|^{2}=r+\left|a_{0}\right|^{2}+\left|c_{1}\right|^{2}, \\
\left|c_{1}\right|^{2}+\left|a_{1}\right|^{2}+\left|b_{2}\right|^{2}=\left|b_{1}\right|^{2}+\left|a_{1}\right|^{2}+\left|c_{2}\right|^{2}, \ldots, \\
\overline{a_{0}} c_{1}+\overline{b_{1}} a_{1}=a_{0} \overline{b_{1}}+c_{1} \overline{a_{1}} \\
\overline{a_{1}} c_{2}+\overline{b_{2}} a_{2}=a_{1} \overline{b_{2}}+c_{2} \overline{a_{2}}, \ldots
\end{gathered}
$$


and

$$
\overline{b_{1}} c_{2}=c_{1} \overline{b_{2}}, \quad \overline{b_{2}} c_{3}=c_{2} \overline{b_{3}}, \ldots
$$

We have denoted $r=\|\xi\|^{2}>0$.

Elementary algebra implies

$$
\left|b_{1}\right|=\left|b_{2}\right|=\left|b_{3}\right|=\cdots ; \quad\left|c_{1}\right|=\left|c_{2}\right|=\left|c_{3}\right|=\cdots .
$$

In virtue of the assumption that the chain of Krylov subspaces $\left(H_{k}\right)$ is not stationary, the entries $c_{k}$ are non-zero.

Then a unitary transformation $T \mapsto U^{*} T U$ with $U$ diagonal reduces lower-diagonal entries to a positive constant:

$$
b_{1}=b_{2}=b_{3}=\cdots=s>0 .
$$

Again simple algebra yields

$$
c_{1}=c_{2}=c_{3}=\cdots=u \in \mathbb{C},
$$

and

$$
a_{1}=a_{2}=a_{3}=\cdots=a
$$

After a translation $T \mapsto T-a I$ and homotethy we can assume $a=0$ and $s=1$ reaching after all the operator

$$
T_{1}=u S+S^{*}
$$

where $S$ denotes the unilateral shift on $\ell^{2}(\mathbb{N})$. But then it is known, and easy to verify, that the principal function $g_{T_{1}}$ is the characteristic function of an ellipse. The affine transformation can be reversed at both levels, operator, respectively, principal function, and thus completes the proof.

We refer to Section 5.3 in [9] for complete details.

The reader will recognize in the above proof a purely matrix algebra phenomenon. To be more specific, let $\mathcal{H}=\left(h_{j k}\right)_{j, k=0}^{\infty}$ denote a Hessenberg matrix with a single non-trivial subdiagonal consisiting of non-null elements. Assume that $\mathcal{H}$ induces a linear bounded operator on $\ell^{2}(\mathbb{N})$ and denote by $\left\{e_{0}, e_{1}, e_{2}, \ldots\right\}$ the canonical orthonormal basis of $\ell^{2}(\mathbb{N})$. Theorem 4.1 states that the apparently innocent conditions

$$
\left[\mathcal{H}, \mathcal{H}^{*}\right]=\alpha e_{0} \otimes e_{0},
$$

and

$$
\mathcal{H}^{*} e_{0}=\beta e_{0}+\gamma e_{1}
$$

where $\alpha>0, \beta, \gamma$ are constants, imply $\mathcal{H}$ is a Toeplitz matrix with at most three non-trivial diagonals: the main diagonal, the first sub-diagonal and the first upper-diagonal. In other terms: $\mathcal{H}=c_{1} S+c_{0}+c_{-1} S^{*}$, where $S$ denotes the unilateral shift and $c_{-1}, c_{0}, c_{1}$ are constants.

A simple adaptation of the commutator identity (4.3) yields the following consequence. 
Corollary 4.2. Let $\left(P_{n}(z)\right)_{n=0}^{\infty}$ be a complete system of exponential orthogonal polynomials associated to a shade function $g$. Let $d$ be a positive integer. If

$$
\int_{\mathbb{C}} z P_{k}(z) g(z) \mathrm{d} A(z)=0, \quad k \geq d+1
$$

then these orthogonal polynomials satisfy a $(d+2)$-recurrence relation:

$$
z P_{k}(z)=\sum_{j=k-d-1}^{k+1} h_{j k} P_{j}(z), \quad k \geq d+1 .
$$

In other terms, if the first row in Hessenberg's matrix $\mathcal{H}$ has non-zero entries only on the positions $0,1, \ldots, d$, then the whole matrix will have only the first $d$ upper-diagonals non-zero. That is $\mathcal{H}$ is a finite banded matrix.

\section{Algebra of exponential transform COEFFicients}

The finite term relation fulfilled by the exponential orthogonal polynomials implies, and can be derived, from purely algebra manipulations of the Taylor coefficients at infinity of the underlying exponential transform. We expand in this section some of these computations bearing a numerical matrix analysis flavor. Seen as a formal transform of the power moments of the generating shade function $g$, the Taylor series at infinity of the exponential transform encodes subtle geometric properties. For instance a specific rationality of this double series is equivalent to $g$ being the characteristic function of a quadrature domain. Along these lines, Chapter 5 of [9] exploits a Padé approximation scheme in two variables of the exponential transform.

Let $T$ be an irreducible hyponormal operator with principal function $g$ and self-commutator $\left[T^{*}, T\right]=\xi \otimes \xi$. An expansion at infinity of the associated exponential transform reads:

$$
\exp \left(-\sum_{j, k=0}^{\infty} \frac{a_{j k}}{z^{j+1} \bar{w}^{k+1}}\right)=1-\sum_{j, k=0}^{\infty} \frac{b_{j k}}{z^{j+1} \bar{w}^{k+1}},
$$

where $\left(a_{j k}\right)$ are the power moments of $g$, while

$$
b_{j k}=\left\langle T^{* k} \xi, T^{* j} \xi\right\rangle, \quad j, k \geq 0 .
$$

In this section we avoid the case of a quadrature domain, when not all polynomials $P_{n}$ are well defined. Recall that

$$
\left\langle P_{j}\left(T^{*}\right) \xi, P_{k}\left(T^{*}\right) \xi\right\rangle=\delta_{j k}
$$

According to Corollary 4.2, a finite term relation holds for the complete system of orthogonal polynomials $\left(P_{j}\right)$ if and only if there exists a positive integer $d$ satisfying

$$
\left\langle T^{*} P_{k}\left(T^{*}\right) \xi, \xi\right\rangle=0, \quad k \geq d+1
$$

or equivalently, the vector $T \xi$ is a linear combination of $\xi, T^{*} \xi, \ldots, T^{* d} \xi$. Let $Q(z)=q_{0}+q_{1} z+\ldots q_{d} z^{d}$ be a polynomial of degree $d$ encoding this dependence:

$$
T \xi=Q\left(T^{*}\right) \xi
$$


In other terms this equality holds if and only if the numerical certificates

$$
b_{m+1,0}=\left\langle T \xi, T^{* m} \xi\right\rangle=\left\langle Q\left(T^{*}\right) \xi, T^{* m} \xi\right\rangle=\sum_{k=0}^{d} q_{k} b_{m, k}, \quad m \geq 0,
$$

are satisfied.

As a matter of fact, if identity (5.2) is true, then the system of exponential orthogonal polynomials is complete. Simply because the vectors $T^{j} T^{* k} \xi, j, k \geq 0$, can be reduced via the commutation relation $\left[T^{*}, T\right]=\xi \otimes \xi$ to combinations of $\xi, T^{*} \xi, T^{* 2} \xi, \ldots$

At the level of formal series, the transfer of these observations to the exponential transform

$$
E(z, w)=1-\sum_{j, k=0}^{\infty} \frac{b_{j k}}{z^{j+1} \bar{w}^{k+1}}
$$

is straightforward. Indeed, the coefficient of $\frac{-1}{z^{m+1} \bar{w}}$ in $z E(z, w)$ is $b_{m+1,0}$, while the same coefficient in $Q(\bar{w}) E(z, w)$ is $\sum_{k=0}^{d} q_{k} b_{m, k}$.

In conclusion, we have proved the following criterion.

Proposition 5.1. Let $\left(P_{n}(z)\right)_{n=0}^{\infty}$ be a complete system of exponential orthogonal polynomials associated to a shade function $g$ and denote by $E(z, w)$ the respective exponential transform. Let d be a positive integer.

The system $\left(P_{n}\right)$ satisfies a $(d+2)$-term relation (4.4) if and only if there exists a polynomial $Q(z)$ of degree $d$ with the property

$$
\operatorname{Res}_{w=0}[(z-Q(\bar{w})) E(z, w)]=0,
$$

identically in $z$.

One step further, we prove in the conditions of the above Proposition that similar recurrence relations fill the full matrix of exponential transform coefficients $\left(b_{j k}\right)_{j, k=0}^{\infty}$ having as input the marginal data $\left(b_{j, 0}\right)_{j=0}^{\infty}$. Indeed, we start from

$$
T^{* n} T \xi=T^{* n} Q\left(T^{*}\right) \xi
$$

where $n$ is a positive integer. The commutation relation imposed on $T$ and $T^{*}$ implies

$$
T^{* n} T=T T^{* n}+\sum_{j=0}^{n-1} T^{* j}\left[T^{*}, T\right] T^{*(n-1-j)}=T T^{* n}+\sum_{j=0}^{n-1} T^{* j} \xi\left\langle\cdot, T^{(n-1-j)} \xi\right\rangle .
$$

Let $m$ be a non-negative integer. Then

$$
\left\langle T T^{* n} \xi, T^{* m} \xi\right\rangle=\left\langle T^{* n} Q\left(T^{*}\right) \xi, T^{* m} \xi\right\rangle-\sum_{j=0}^{n-1}\left\langle T^{* j} \xi, T^{* m} \xi\right\rangle\left\langle\xi, T^{(n-1-j)} \xi\right\rangle .
$$

Hence we can state the following complement to the finite term criterion. 
Corollary 5.2. Let $\left(P_{n}(z)\right)_{n=0}^{\infty}$ be a complete system of exponential orthogonal polynomials associated to a shade function $g$ and denote by

$$
E(z, w)=1-\sum_{j, k=0}^{\infty} \frac{b_{j k}}{z^{j+1} \bar{w}^{k+1}}
$$

the respective exponential transform. Fix a positive integer $d$.

If there are complex constants $q_{0}, q_{1}, \ldots, q_{d}$ satisfying

$$
b_{m+1,0}=\sum_{k=0}^{d} q_{k} b_{m, k}, \quad m \geq 0,
$$

then

$$
b_{m+1, n}=\sum_{k=0}^{d} q_{k} b_{m, k+n}-\sum_{j=0}^{n-1} b_{m j} b_{0, n-j-1},
$$

for all $m \geq 0$ and $n \geq 1$.

In function theory terms, we rephrase the above computations into the following statements invoking a shade function $g$ which carries a complete system of exponential orthogonal polynomials:

(1). Fix a positive integer $d$. The Cauchy transforms

$$
F_{k}(z)=\frac{-1}{\pi} \int \frac{g(\zeta) \bar{\zeta}^{k} \mathrm{~d} A(\zeta)}{\zeta-z}, 0 \leq k \leq d
$$

determine via condition (5.4) whether the exponential orthogonal polynomials satisfy a $(d+2)$-finite term relation.

To be more precise, we seek a polynomial $Q(z)$ of degree $d$ with the property

$$
\operatorname{Res}_{w=0}\left[(z-Q(\bar{w})) \exp \left(-\frac{F_{0}(z)}{\bar{w}}-\frac{F_{1}(z)}{\bar{w}^{2}}-\cdots-\frac{F_{d}(z)}{\bar{w}^{d+1}}\right)\right]=0, \quad|z| \gg 1
$$

(2). In this case, Cauchy's transform $F_{0}(z)$ and the polynomial $Q(z)$ resulting from 1) determine the generating shade function $g$.

Notice that condition (5.7) does not imply, in general, that the system of exponential orthogonal polynomials is complete. Rotationally invariant shapes, discussed in Section 7 provide counterexamples.

The numerical analyst might feel betrayed by statement (2) above. A clarification is in order: our recursion formulas (5.6) determine all power moments of the function $g$. In particular one can estimate from there the location of the support of $g$, say in the unit square $-1 \leq \operatorname{Re} z \leq 1,-1 \leq \operatorname{Im} z \leq 1$. Then $g$ (a bounded measurable function) can be recovered by its Fourier expansion with respect to a basis of classical bi-variate orthogonal polynomials adapted to the square (such as Legendre polynomials), or via an inversion formula for the Laplace-Fourier transform of $g$. We do not expand here these details. 


\section{EXAMPLES}

In this section we illustrate by means of a couple of examples a departure from the Bergman or Hardy space scenario. To be more specific, a finite term relation for the exponential orthogonal polynomials does not imply in general that the underlying shape is an ellipse. On the contrary, both Bergman orthogonal polynomials or Szegö orthogonal polynomials (i.e. with respect to arc length of a closed contour) imply that the original domain is an ellipse, $c f .[6,13,17,20]$.

\subsection{Two non-zero diagonals}

We will construct a sparse infinite matrix $T$, bounded as a linear transformation on $\ell^{2}(\mathbb{N})$ and satisfying

$$
\left[T^{*}, T\right]=e_{0} \otimes e_{0},
$$

where $\left\{e_{0}, e_{1}, e_{2}, \ldots\right\}$ is the orthonormal basis with respect to which one expresses the matrix elements.

Specifically, we choose recursively real numbers $a_{1}, a_{2}, a_{3}, \ldots$ and $b_{0}, b_{1}, b_{2}, \ldots$ which populate the matrix

$$
T=\left(\begin{array}{ccccc}
0 & a_{1} & 0 & 0 & \ldots \\
0 & 0 & a_{2} & 0 & \ldots \\
b_{0} & 0 & 0 & a_{3} & \\
0 & b_{1} & 0 & 0 & \ddots \\
\vdots & & \vdots & & \ddots
\end{array}\right) .
$$

We display for convenience the adjoint:

$$
T^{*}=\left(\begin{array}{ccccc}
0 & 0 & b_{0} & 0 & \ldots \\
a_{1} & 0 & 0 & b_{1} & \ldots \\
0 & a_{2} & 0 & 0 & b_{2} \\
0 & 0 & a_{3} & 0 & \ddots \\
\vdots & & \vdots & & \ddots
\end{array}\right)
$$

Condition (6.1) is equivalent to

$$
\begin{gathered}
b_{0}^{2}-a_{1}^{2}=1, \\
a_{1}^{2}+b_{1}^{2}=a_{2}^{2}, \\
a_{2}^{2}+b_{2}^{2}=a_{3}^{2}+b_{0}^{2}, \\
a_{n}^{2}+b_{n}^{2}=a_{n+1}^{2}+b_{n-2}^{2}, \quad n \geq 2,
\end{gathered}
$$

and

$$
\begin{gathered}
b_{0} a_{3}=a_{1} b_{1}, \\
b_{1} a_{4}=a_{2} b_{2}, \\
b_{n-1} a_{n+2}=a_{n} b_{n}, \quad n \geq 1 .
\end{gathered}
$$


We denote for convenience $A_{n}=a_{n}^{2}, \quad n \geq 1$, and $B_{n}=b_{n}^{2}, \quad n \geq 0$. Therefore

$$
B_{0}=A_{1}+1, B_{1}=A_{2}-A_{1}
$$

and in general

$$
B_{n}=A_{n+1}-A_{n}+B_{n-2}, \quad n \geq 2 .
$$

Similarly,

$$
B_{n-1} A_{n+2}=A_{n} B_{n}, \quad n \geq 1 .
$$

Note that addition yields

$$
B_{n}+B_{n-1}=A_{n+1}+1, \quad n \geq 1 .
$$

We first prove that by selecting the initial data $A_{1}>0, B_{1}>0$ the double sequence recurrence goes through to infinity. Remark that $A_{3}>0$ in this case.

Indeed, since division is involved we have to check that all $A_{k}^{\prime} s$ are non-zero. Assume $A_{k+3}=0$ and this is the first occurrence (for some $k>0$ ). We infer from

$$
A_{k+3} B_{k}=A_{k+1} B_{k+1}=0
$$

that $B_{k+1}=0$. But then

$$
A_{k+4} B_{k+1}=A_{k+2} B_{k+2}=0
$$

implies $B_{k+2}=0$. In conclusion we obtain a finite matrix

$$
S=\left(\begin{array}{cccccc}
0 & a_{1} & 0 & 0 & \ldots & 0 \\
0 & 0 & a_{2} & 0 & \ldots & 0 \\
b_{0} & 0 & 0 & a_{3} & \ldots & 0 \\
\vdots & & \vdots & & \ddots & \vdots \\
0 & \ldots & b_{n-1} & 0 & 0 & a_{n+2} \\
0 & \ldots & 0 & b_{n} & 0 & 0
\end{array}\right)
$$

which satisfies $\left[S^{*}, S\right]=e_{0} \otimes e_{0}$. But this is a contradiction to the fact that a commutator of finite matrices has vanishing trace. Or simply remark that identity (6.2) is violated.

Thus the recurrence goes through to infinity and produces the two sequences of real numbers $\left(a_{n}\right),\left(b_{n}\right)$ and respectively the sequences of positive numbers $\left(A_{n}\right),\left(B_{n}\right)$.

Returning to the generating identities, we can eliminate $B_{n}$ by multiplying (6.2) by $A_{n+2}$ :

$$
B_{n} A_{n+2}+B_{n} A_{n}=A_{n+2}+A_{n+2} A_{n+1}
$$

whence

$$
B_{n}=\frac{A_{n+2}+A_{n+2} A_{n+1}}{A_{n}+A_{n+2}}, \quad n \geq 1 .
$$


On the other hand,

$$
B_{n}=\frac{A_{n+1}}{A_{n+3}} B_{n+1}
$$

yields

$$
A_{n+2}\left(1+A_{n+1}\right) A_{n+3}\left(A_{n+1}+A_{n+3}\right)=A_{n+1} A_{n+3}\left(1+A_{n+2}\right)\left(A_{n+1}+A_{n+3}\right),
$$

or

$$
\frac{A_{n+3}+A_{n+1}}{A_{n+2}+A_{n}}=\frac{1+1 / A_{n+2}}{1+1 / A_{n+1}}, \quad n \geq 1
$$

The telescopic product implies

$$
A_{n+3}+A_{n+1}=\frac{A_{3}+A_{1}}{1+1 / A_{2}}\left(1+1 / A_{n+2}\right), \quad n \geq 0 .
$$

Denote $C=\frac{A_{3}+A_{1}}{1+1 / A_{2}}$.

It remains to prove that the sequence $\left(A_{n}\right)$ is uniformly bounded from above. In virtue of (6.2) the sequence $\left(B_{n}\right)$ would also be bounded and therefore $T$ will be a linear continuous transformation on $\ell^{2}$.

Assume that there exists a subsequence $A_{n(j)}$ satisfying

$$
\lim _{j} A_{n(j)}=\infty .
$$

Identity

$$
C\left(1+\frac{1}{A_{n(j)+1}}\right)=A_{n(j)}+A_{n(j)+2}
$$

yields

$$
\lim _{j} A_{n(j)+1}=0 .
$$

Thus $B_{n(j)-1}+B_{n(j)}=A_{n(j)+1}+1$ remains bounded as a function of $j$. From the similar identity $B_{n(j)-2}+B_{n(j)-1}=A_{n(j)}+1$ we find

$$
\lim _{j} B_{n(j)-2}=\infty
$$

And $B_{n(j)-3}+B_{n(j)-2}=A_{n(j)-1}+1$ implies

$$
\lim _{j} A_{n(j)-1}=\infty
$$

By repeating the above reasoning we find

$$
\lim _{j} A_{n(j)}=0
$$


a contradiction.

Summing up, the following statement was just proved.

Proposition 6.1. There exists a Borel measurable shade function $g: \mathbb{C} \longrightarrow[0,1]$ with compact support, so that the associated exponential orthogonal polynomials $P_{n}(z)$ satisfy the recursion

$$
z P_{n}(z)=a_{n+1} P_{n+1}(z)+b_{n-2} P_{n-2}(z), \quad n \geq 0
$$

where $P_{-1}=P_{-2}=0$.

In this case $g$ is not the characteristic function of an ellipse, nor a quadrature domain.

\subsection{Toeplitz operators}

If we do not insist on only two non-zero diagonals, one can produce a similar counterexample as follows. Denote as before by $S$ the unilateral shift acting on $\ell^{2}(\mathbb{N})$. Consider the linear operator $T=S^{2}+S^{*}$. One verifies directly, for instance evaluating on the orthonormal basis $\left\{e_{0}, e_{1}, e_{2}, \ldots\right\}$, that $\left[S^{*}, S\right]=e_{0} \otimes e_{0}$ and

$$
\left[S^{* 2}, S^{2}\right]=e_{0} \otimes e_{0}+e_{1} \otimes e_{1} .
$$

Hence

$$
\left[T^{*}, T\right]=\left[S^{* 2}, S^{2}\right]+\left[S, S^{*}\right]=e_{1} \otimes e_{1}
$$

Thus $T$ is a hyponormal operator with rank-one self-commutator. And it satisfies our finite term relation constraint. Indeed,

$$
T e_{1}=e_{3}+e_{0},
$$

and

$$
T^{*} e_{1}=e_{2}, \quad T^{*} e_{2}=e_{0}+e_{3} .
$$

That is

$$
T e_{1}=T^{* 2} e_{1} .
$$

One checks by recurrence that for all $k \geq 0$, the vectors $e_{3 k+1}, e_{3 k+2}, e_{3 k}+e_{3 k+3}$ belong to the polynomial closure subspace $H_{\text {pol }}$. We infer that the vectors $T^{* n} e_{1}, n \geq 0$, generate the space $\ell^{2}(\mathbb{N})$, which means that $T$ is an irreducible hyponormal operator and the exponential orthogonal polynomials are complete.

Since $T$ is a Toeplitz operator with compact self-commutator, its essential spectrum coincides with the real algebraic curve

$$
\Gamma=\left\{z^{2}+\frac{1}{z} ;|z|=1\right\}
$$

and the spectrum of $T$ is the bounded set surrounded by $\Gamma$. As a matter of fact $\Gamma$ is a cycloid, in the form of three leaves, with a $2 \pi / 3$ rotational symmetry. Indeed, writing $z=e^{i \theta}$ for a point on the circle, the curve $\Gamma$ is described by the parametric equations

$$
x=\cos 2 \theta+\cos \theta, \quad y=\sin 2 \theta-\sin \theta,
$$


or better for visualization:

$$
x=2 \cos \frac{3 \theta}{2} \cos \frac{\theta}{2}, \quad y=2 \cos \frac{3 \theta}{2} \sin \frac{\theta}{2},
$$

whence, in polar coordinates:

$$
r=2\left|\cos \frac{3 \theta}{2}\right|,
$$

where $\theta \in[0,2 \pi]$.

In conclusion the spectrum of the Toeplitz operator $T$ is a trifoil, with 3 -fold rotational symmetry, consisting of three leaves intersecting at the origin. Their interiors are disjoint and carry a non-essential spectrum of Fredholm index equal to -1 .

The exponential orthogonal polynomials $P_{n}, n \geq 0$, associated to $T$ satisfy therefore a four term relation, propagated from the first row $T e_{1}=e_{3}+e_{0}=T^{* 2} e_{1}$, that is:

$$
z P_{k}=h_{k+1, k} P_{k+1}+h_{k, k} P_{k}+h_{k-1, k} P_{k-1}+h_{k-2, k} P_{k-2}, \quad k \geq 2 .
$$

Note that the associated Hessenberg matrix carries four non-zero diagonals, while the Toeplitz matrix representation of the same operator has only two non-zero diagonals.

Note that one can equally consider the family of Toeplitz operators

$$
\alpha S^{2}+\beta S^{*}, \quad|\alpha|=|\beta|>0 \text {. }
$$

If only aiming at producing a Toeplitz operator with rank-one self-commutator, one can look at

$$
V=\alpha S^{d+1}+\beta S^{* d}, \quad|\alpha|=|\beta|>0,
$$

where $d$ is a fixed positive integer. One proves as before that

$$
\left[V^{*}, V\right]=e_{d} \otimes e_{d}
$$

Since

$$
V e_{d}=e_{2 d+1}+e_{0}
$$

and

$$
\left(V^{*}\right)^{N} e_{d}=e_{(N+1) d}+\text { lower order terms, } \quad N \geq 0,
$$

we observe that in case $d>1$ there is no polynomial $G \in \mathbb{C}[z]$ with the property

$$
V e_{d}=G\left(V^{*}\right) e_{d}
$$

Therefore, in this case, the Hessenberg matrix associated to the exponential orthogonal polynomials is not finitely banded. 


\section{ROTATIONALLY INVARIANT SHAPES}

In this section we specialize our analysis to the case of a rotationally invariant principal function

$$
g(\zeta)=g_{T}(\zeta)=g_{T}(|\zeta|), \quad \zeta \in \mathbb{C}
$$

In terms of the associate hyponormal operator $T$, this invariance means that for every $\theta \in[0,2 \pi)$ there exists a unitary transformation $U_{\theta}: H \longrightarrow H$ satisfying

$$
e^{i \theta} T=U_{\theta} T U_{\theta}^{*}
$$

Under this assumption the exponential transform simplifies:

$$
\begin{aligned}
E_{g}(z, w) & =\exp \left[\frac{-1}{\pi} \int \frac{g(r) r \mathrm{~d} r \mathrm{~d} \theta}{\left(r e^{i \theta}-z\right)\left(r e^{-i \theta}-\bar{w}\right)}\right] \\
& =\exp \left[\int_{0}^{\infty} \frac{g(r) \mathrm{d}\left(r^{2}\right)}{r^{2}-z \bar{w}}\right]=\exp \left[\int_{0}^{\infty} \frac{g(\sqrt{t}) \mathrm{d} t}{t-z \bar{w}}\right] .
\end{aligned}
$$

These formulas, obtained via power expansion at infinity, are valid for large values of $|z|$ and $|w|$. The additive representation of analytic functions with positive imaginary part in the upper half plane [2] yields a positive measure $\nu$ supported by the semiaxis, with the property:

$$
\exp \left[\int_{0}^{\infty} \frac{g(\sqrt{t}) \mathrm{d}(t)}{t-z \bar{w}}\right]=1+\int_{0}^{\infty} \frac{\mathrm{d} \nu(s)}{s-z \bar{w}}
$$

Since the original "phase shift" $g(\sqrt{t})$ has compact support, the representing measure $\nu$ is also compactly supported. And vice-versa, any such measure corresponds to a $g(\sqrt{t})$, see again [2].

On the other hand, the pure hyponormal operator $T$ with principal function equal to $T$ satisfies:

$$
1-\left\langle\left(T^{*}-\bar{w}\right)^{-1} \xi,\left(T^{*}-\bar{z}\right)^{-1} \xi\right\rangle=1+\int_{0}^{\infty} \frac{\mathrm{d} \nu(s)}{s-z \bar{w}}
$$

hence

$$
-\sum_{k=0}^{\infty} \frac{\left\|T^{* k} \xi\right\|^{2}}{z^{k+1} \bar{w}^{k+1}}=\int_{0}^{\infty} \frac{\mathrm{d} \nu(s)}{s-z \bar{w}}
$$

From here we identify $\left\|T^{* k} \xi\right\|^{2}, k \geq 0$, with the Stieltjes sequence of moments of a positive measure with compact support on the semi-axis. And conversely, a compactly supported Stieltjes measure $\nu$ gives rise through the above formulas to a unique rotationally invariant irreducible hyponormal operator $T$ with rank-one self-commutator.

For instance the unit disk carrying the Lebesgue measure $\left(g=\chi_{\mathbb{D}} \mathrm{d} A\right)$ corresponds to the Dirac mass $\nu=\delta_{0}$. Notice that in this case $T^{*} \xi=0$ and it is the only situation when the space

$$
H_{\mathrm{pol}}=\bigvee_{n \geq 0} T^{* n} \xi
$$

is finite dimensional. 
Assuming the space $H_{\text {pol }}$ is infinite dimensional, we turn to the associated exponential orthogonal polynomials. Due to the orthogonality conditions

$$
\left\langle T^{* k} \xi, T^{* m} \xi\right\rangle=0, \quad k \neq m,
$$

these are precisely

$$
P_{n}(z)=\frac{z^{n}}{\left\|T^{* n} \xi\right\|^{1 / 2}}, \quad n \geq 0 .
$$

Thus the associated Hessenberg matrix $\mathcal{H}$ has only the first subdiagonal non-zero, say

$$
\mathcal{H}=\left(\begin{array}{ccccc}
0 & 0 & 0 & 0 & \ldots \\
\gamma_{0} & 0 & 0 & 0 & \ldots \\
0 & \gamma_{1} & 0 & 0 & \ldots \\
0 & 0 & \gamma_{2} & 0 & \ddots \\
\vdots & & \vdots & & \ddots
\end{array}\right)
$$

where $\gamma_{k}>0$ for all $k \geq 0$.

If in addition to the subspace $H_{\text {pol }}$ being infinite dimensional it coincides with the full Hilbert space $H$, then $\mathcal{H}$ represents $T^{*}$ with respect to the basis $e_{n}=P_{n}\left(T^{*}\right) \xi$. But since $\left[T^{*}, T\right]=\xi \otimes \xi$ this gives

$$
-\gamma_{0}^{2}=\frac{\left\langle\left[T^{*}, T\right] \xi, \xi\right\rangle}{\|\xi\|^{2}}>0
$$

a contradiction.

In short we have proved that for rotationally invariant hyponormal operators in our class, the space $H_{\text {pol }}$ spanned by the exponential orthogonal polynomials never coincides with the full space $H$. We elaborate two simple examples.

Example 7.1. Let $g=t \chi_{\mathbb{D}}$ for some $0<t<1$. Then

$$
E_{g}(z, w)=1-\left\langle\left(T^{*}-\bar{w}\right)^{-1} \xi,\left(T^{*}-\bar{z}\right)^{-1} \xi\right\rangle
$$

is to be identified with

$$
\left(E_{\mathbb{D}}(z, w)\right)^{t}=\left(1-\frac{1}{z \bar{w}}\right)^{t}
$$

for large $z, w$. This gives, after power expansions,

$$
\begin{gathered}
\left\langle T^{* k} \xi, T^{* k} \xi\right\rangle=(-1)^{k}\left(\begin{array}{c}
t \\
k+1
\end{array}\right)=\frac{t(1-t) \ldots(k-t)}{(k+1) !}, \\
\left\langle T^{* k} \xi, T^{* j} \xi\right\rangle=0, \quad k \neq j .
\end{gathered}
$$

Denoting

$$
c_{k}=1 / \sqrt{(-1)^{k}\left(\begin{array}{c}
t \\
k+1
\end{array}\right)}>0
$$


we obtain the canonical ON-basis $e_{0}, e_{1}, e_{2}, \ldots$ of the space $H_{\text {pol }}$ given by

$$
\left\{\begin{array}{l}
e_{0}=c_{0} \xi \\
e_{1}=c_{1} T^{*} \xi \\
e_{2}=c_{2} T^{* 2} \xi \\
e_{3}=c_{3} T^{* 3} \xi \\
\cdots
\end{array}\right.
$$

In terms of $\left\{e_{0}, e_{1}, e_{2}, \ldots\right\}$ the compression to the polynomial subspace of the cohyponormal operator $T^{*}$ is represented by Hessenberg's matrix

$$
\mathcal{H}=\left(\begin{array}{ccccc}
0 & 0 & 0 & 0 & \ldots \\
c_{0} / c_{1} & 0 & 0 & 0 & \ldots \\
0 & c_{1} / c_{2} & 0 & 0 & \ldots \\
0 & 0 & c_{2} / c_{3} & 0 & \ddots \\
\vdots & & \vdots & & \ddots
\end{array}\right)
$$

However this compression is not hyponormal itself, only the full operator is.

To find the measure $\nu$ in the additive representation of the Nevanlinna analytic function

$$
f(z)=\left(1-\frac{1}{z}\right)^{t}, \quad z \notin[0,1]
$$

we have to compute, at the level of distributions

$$
\pi \nu(x)=\lim _{\epsilon \rightarrow 0} \operatorname{Im} f(x+i \epsilon)
$$

see [2]. In the limit $t=1$ this gives $\nu=\delta_{0}$, as expected, while for $0<t<1 \nu$ is absolutely continuous with density

$$
\frac{\mathrm{d} \nu}{\mathrm{d} x}=\frac{\sin (\pi t)}{\pi}\left(\frac{1}{x}-1\right)^{t}, \quad 0<x<1
$$

Example 7.2. The annulus also offers another computable example. Specifically, for $g=\chi_{\mathbb{D}_{R}}-\chi_{\mathbb{D}_{r}}, 0<r<R$, we find:

$$
\begin{aligned}
E_{g}(z, w) & =\frac{E_{\mathbb{D}_{R}}(z, w)}{E_{\mathbb{D}_{r}}(z, w)}=\frac{1-\frac{R^{2}}{z \bar{w}}}{1-\frac{r^{2}}{z \bar{w}}}=\left(1-\frac{R^{2}}{z \bar{w}}\right)\left(1+\frac{r^{2}}{z \bar{w}}+\frac{r^{4}}{z^{2} \bar{w}^{2}}+\ldots\right) \\
& =1-\left(R^{2}-r^{2}\right)\left(\frac{1}{z \bar{w}}+\frac{r^{2}}{z^{2} \bar{w}^{2}}+\frac{r^{4}}{z^{3} \bar{w}^{3}}+\ldots\right)
\end{aligned}
$$

to be identified with

$$
1-\left\langle\left(T^{*}-\bar{w}\right)^{-1} \xi,\left(T^{*}-\bar{z}\right)^{-1} \xi\right\rangle=-\sum_{k, j \geq 0} \frac{\left\langle T^{* j} \xi, T^{* k} \xi\right\rangle}{z^{k+1} \bar{w}^{j+1}} .
$$


It follows that the canonical ON-basis is

$$
\left\{\begin{array}{l}
e_{0}=\frac{1}{\sqrt{R^{2}-r^{2}}} \xi \\
e_{1}=\frac{1}{r \sqrt{R^{2}-r^{2}}} \xi \\
e_{2}=\frac{1}{r^{2} \sqrt{R^{2}-r^{2}}} \xi \\
e_{3}=\frac{1}{r^{3} \sqrt{R^{2}-r^{2}}} \xi \\
\ldots,
\end{array}\right.
$$

with the compression of $T^{*}$ to the polynomial subspace $H_{\text {pol }}$ represented by the matrix

$$
\mathcal{H}=\left(\begin{array}{ccccc}
0 & 0 & 0 & 0 & \ldots \\
1 / r & 0 & 0 & 0 & \ldots \\
0 & 1 / r & 0 & 0 & \ldots \\
0 & 0 & 1 / r & 0 & \ddots \\
\vdots & & \vdots & & \ddots
\end{array}\right)
$$

In this case the additive representation of the corresponding Nevanlinna function

$$
f(z)=\frac{z-R^{2}}{z-r^{2}}
$$

is straightforward:

$$
f(z)=1+\int_{\mathbb{R}} \frac{\mathrm{d} \nu(s)}{s-z}
$$

where $\mathrm{d} \nu=\left(R^{2}-r^{2}\right) \delta_{r^{2}}$.

We also validate in this particular situation the general fact that Hessenberg matrix $\mathcal{H}$ together with the positive number $\|\xi\|^{2}=\frac{R^{2}-r^{2}}{\pi}$ determines the original shape. Obviously knowledge of the norm of $\xi$ is really needed here since the matrix (7.1) gives no information about the radius $R$.

Related to the latter statement is the fact that $H_{\text {pol }}$ is strictly smaller than $H$, despite it being infinite dimensional. In fact, if the two spaces had been equal, then $\mathcal{H}$ would have fully represented $T^{*}$ and all information of $\xi$ would have been built into it. In addition, $\mathcal{H}$ would have been cohyponormal, while the matrix in (7.1) clearly is (strictly) subnormal (and hyponormal).

One can see these features clearly in functional models of $H$, discussed in $[9,10]$. Such models are generated by the monomials $z^{k} \bar{z}^{j}, k, j \geq 0$, considered as functions defined on the spectrum of the operator. But these monomials are far from being linearly independent in general. For example, when $\operatorname{dim} H_{\text {pol }}<\infty$ then there is a monic (say) polynomial of $P_{d}$ of degree $d=\operatorname{dim} H_{\text {pol }}$ such $P_{d}\left(T^{*}\right) \xi=0$ (cf. Sect. 3), which means that the relation $P_{d}(z)=0$ holds in the relevant functional space. In this case int $\sigma$ is a quadrature domain with quadrature nodes at the zeros of $P_{d}$.

When $\sigma$ is the characteristic function of an ellipse it turns out that a relation $\bar{z}=a z$ holds for some constant $a \in \mathbb{C}$ (see [10], Example 4.2), and as for the present example, with the annulus, we have a relation

$$
\bar{z}=\frac{R^{2}+r^{2}}{2 z} .
$$


As explained in [10] (Thm. 4.1 there) such a relation comes out on exhibiting a continuous function $f$ in $\mathbb{C}$ which vanishes outside $\sigma$ and which in int $\sigma$ satisfies

$$
\frac{\partial f}{\partial \bar{z}}=\bar{z}-\frac{R^{2}+r^{2}}{2 z}
$$

In the present case this function is

$$
f(z)=\frac{\left(|z|^{2}-r^{2}\right)\left(\left|z^{2}\right|-R^{2}\right)}{2 z^{2}} .
$$

The relation (7.2) means that instead of letting the functional space be generated by the two parameter family $z^{k} \bar{z}^{j}(k, j \geq 0)$, it is enough to use the sequence $z^{n}$, with $n$ running through all integers (positive and negative). And these functions are then linearly independent. This is in full agreement with the general result ([9], Thm. 3.1) saying that the analytic functions on $\sigma$ are densely injected in the functional model of $H$, in the non-quadrature domain case.

\section{Harmonic moments and Hele-Shaw flow}

The first column of the power moments, $\left\{a_{j 0}, j \geq 0\right\}$, namely the "harmonic" moments, coincides with the first column of the exponential moments, $\left\{b_{j 0}, j \geq 0\right\}$, as is easily seen from (5.1). Hele-Shaw flow moving boundary problems, or Laplacian growth (see $[11,22]$ for general theory), are characterized by these moments changing according to some simple law. One case is the squeezing version of Hele-Shaw flow, meaning that a viscous fluid blob, represented by the principal function $g$, is confined between two parallel plates and one simply squeezes the plates together. The dynamics is then that the harmonic moments decrease uniformly with respect to time $t$, say as

$$
a_{j 0}(t)=b_{j 0}(t)=C e^{-t}, \quad 0 \leq t<\infty .
$$

Of major interest is also the inverse process, letting $t \rightarrow-\infty$ above whenever this is possible. This backward evolution is highly unstable, but when it is successful it leads in the limit to potential theoretic skeletons, or "mother bodies", as discussed for example in $[9,18]$.

It is quite reasonable to consider principal functions $g=g(t)$ of fairly general form (subject only to $0 \leq g \leq 1$ ). Indeed, the equations describing Hele-Shaw flow are equivalent to those describing porous medium flow, namely Darcy's law, and if $0<g<1$ in some region it simply means that the porous medium is not fully saturated with fluid there. Compare Example 7.1.

However, the harmonic moments describe the dynamics accurately only when $g$ is of the form $\chi_{\Omega}$ with $\Omega$ a simply connected domain, otherwise they do not characterize $g$ completely. With $g$ of this form, the most studied version of Hele-Shaw flow is that of injection or suction of fluid at one point, say the origin. In that case the dynamical law is that $a_{00}$ changes linearly with time, while $a_{10}, a_{20}, \ldots$ remain fixed. This fits well into the recursion algorithm in Corollary 5.2 since the left member $b_{m+1,0}=a_{m+1,0}$ in (5.5) then will be conserved in time, as well as the factors $b_{0, n-j-1}=a_{0, n-j-1}$ in (5.6) (for $0 \leq j \leq n-2$ ).

It is however not clear at present how much of help this is for constructing solutions. Knowledge of all the $b_{k j}$ is equivalent to knowledge of the complete exponential transform, and with the exponential transform at hand the boundary of the fluid domain is immediately obtained as $\partial \Omega=\{z \in \mathbb{C}: E(z, z)=0\}$. Here $E(z, w)$ refers to the restriction of the exponential transform to the complement of the spectrum $\bar{\Omega}$ and the analytic continuation of that across $\partial \Omega$ (known to exist in the present situation). But the step from the first column of $\left(b_{k j}\right)$ to the full matrix is a major challenge (and possible in principle only for $g$ of the form $\chi_{\Omega}$ with $\Omega$ simply connected).

One may also consider Hele-Shaw flow in exterior domains, containing the point of infinity. In this case one usually considers suction at infinity, which means, in our setting, that the spectrum $\sigma$ of the hyponormal 
operator grows. This spectrum, which hence is the complement of the fluid region in the Hele-Shaw model, may represent, in other physical and mathematical models, an electronic droplet in a Coulomb gas or a quantum Hall regime, an aggregate of eigenvalues for a normal random matrix ensemble, a growing crystal in a phase transition model, occupied sites for DLA (diffusion limited aggregation), etc. These subjects also connect to theories of integrable hierarchies, and here a superficial similarity with the material of the present paper is the occurrence of a pair of "Lax" operators, adjoint to each other and satisfying a "string equation", actually a form of Heisenberg's uncertainty relation, which reminds of our commutation relation (2.1). In addition, the Lax operators are naturally represented by Hessenberg matrices. We refer to [16, 21, 23] for some details on the above matters.

In the above inverse geometry, having the Hele-Shaw fluid in the complement of the spectrum, the usual harmonic moments $a_{j 0}$ are no longer preserved. Instead it is the harmonic moments of the exterior domain that behave well. In the notation of the mentioned papers related to integrable hierarchies these are

$$
t_{k}=\frac{1}{2 \pi i k} \int_{\partial \sigma} z^{-k} \bar{z} \mathrm{~d} z \quad k=1,2,3, \ldots
$$

and they are interpreted as "generalized times". Ordinary time is then the moment $t=t_{0}=a_{00}$.

A good example is the ellipse, which is singled out in Theorem 4.1 as the unique configuration admitting a three term relation for the exponential polynomials. An ellipse is characterized by 5 real parameters, and these can be taken to be $t_{0}, t_{1}, t_{2}\left(t_{0}\right.$ is necessarily real). The remaining exterior moments vanish: $t_{3}=t_{4}=\cdots=0$ for any ellipse.

In the Hele-Shaw problem the ellipse shows up as a preserved shape in two ways. One is with having the fluid in the exterior domain, with injection or suction at infinity. This exterior problem means that the boundary moves with speed proportional to the density of the equilibrium measure (for the interior domain), and in the ellipse case, taking the ellipses to be centered at the origin for simplicity, the resulting dynamics will be a family of homothetic ellipses, defined via scale change in the complex plane. Under the evolution $t_{0}$ equals the area of the ellipse divided by $\pi$, while $t_{1}$ and $t_{2}$ remain fixed (like $t_{3}=t_{4}=\cdots=0$ of course). This property of homothetic ellipses is related to the discovery of Newton that the shell between two such ellipses produces no gravitational field in the cavity inside the shell.

When the fluid is inside the ellipse it is the squeezing version of Hele-Shaw flow which preserves the elliptic shape. The family of ellipses is however different, this time it is the family of confocal ellipses, i.e., the oneparameter family obtained by keeping the foci fixed. This is one of few cases in which the dynamics of the squeezing problem works out well in the full range $-\infty<t<\infty$. In the limit $t \rightarrow-\infty$ one obtains the (unique in this case) mother body, which is a measure supported by the segment between the foci. In the standard case with the foci at \pm 1 the density of the measure with respect to arclength along the $x$-axis is proportional to $\sqrt{1-x^{2}}$. For details on the above statements for ellipses we refer to $[12,19,22]$.

It remains open how the three term relation for ellipses fits into the above pictures, but it has at least been proved that the zeros of the exponential polynomials are attracted by the mother body: the counting measures of the zeros have a weak star limit (as the degrees of the polynomials tend to infinity), and this is a measure which has the same support as the mother body (however with a different density). See Proposition 7.1 in [9].

\section{REFERENCES}

[1] D. Aharonov and H.S. Shapiro, Domains on which analytic functions satisfy quadrature identities. J. Anal. Math 30 (1976) $39-73$.

[2] N. Aronsajn and W.F. Donoghue, On exponential representations of analytic functions in the upper half-plane with positive imaginary part. J. Analyse Math. 5 (1956) 321-388.

[3] B. Beckermann, Complex Jacobi Matrices. J. Comp. Appl. Math. 127 (2001) 17-65.

[4] A. Böttcher and S.M. Grudsky, Spectral Properties of Banded Toeplitz Matrices, SIAM, Philadelphia, 2005.

[5] C.F. Dunkl and Y. Xu, Orthogonal Polynomials of Several Variables, Vol. 155 of Encyclopedia of Mathematics and its Applications, Second Edition. Cambridge Univ. Press, Cambridge (2014).

[6] P. Duren, Polynomials orthogonal over a curve. Michigan Math. J. 12 (1965) 313-316. 
[7] J. Favard, Sur les polynomes de Tchebicheff. C. R. Acad. Sci. Paris 200 (1935) 2052-2053.

[8] B. Gustafsson and M. Putinar, The exponential transform: a renormalized Riesz potential at crirical exponent. Indiana Univ. Math. J. 52 (2003) 527-568.

[9] B. Gustafsson and M. Putinar, Hyponormal Quantization of Planar Domains. Vol. 2199 of Lect. Notes Math. Springer, Cham, Switzerland (2017).

[10] B. Gustafsson and M. Putinar, A field theoretic operator model and Cowen-Douglas class. Banach J. Math. 13 (2019) $338-358$.

[11] B. Gustafsson, R. Teoderscu and A. Vasil'ev, Classical and Stochastic Laplacian growth, Advances in Mathematical Fluid Mechanics. Birkhäuser Verlag, Basel (2014).

[12] D. Khavinson and E. Lundberg, Linear Holomorphic Partial Differential Equations and Classical Potential Theory. Vol. 232 of Mathematical Surveys and Monographs. American Mathematical Society, Providence, RI (2018).

[13] D. Khavinson and N. Stylianopoulos, Recurrence relations for orthogonal polynomials and algebraicity of solutions of the Dirichlet problem. Around the Research of Vladimir Maz'ya II S. Partial Differential Equations. Springer, Berlin (2009) $219-228$.

[14] F. Marcellán and R. Álvarez-Nodarse, On the "Favard Theorem" and its extensions. J. Computat. Appl. Math. 127 (2001) $231-254$.

[15] M. Martin and M. Putinar, Lectures on Hyponormal Operators. Birkhäuser Verlag, Basel (1989).

[16] M. Mineev-Weinstein, M. Putinar and R. Teodorescu, Random matrices in 2D, Laplacian growth and operator theory. J. Phys. A 41 (2008) 1-74.

[17] M. Putinar and N. Stylianopoulos, Finite-term relations for planar orthogonal polynomials. Compl. Anal. Oper. Theory 1 (2007) 447-456.

[18] T. Savina, B. Sternin and V. Shatalov, On a minimal element for a family of bodies producing the same external gravitational field. Appl. Anal. 84 (2005) 649-668.

[19] H.S. Shapiro, The Schwarz Function and its Generalization to Higher Dimensions. In Vol. 9 of University of Arkansas Lecture Notes in the Mathematical Sciences. John Wiley \& Sons Inc., New York (1992).

[20] G. Szegö, A problem concerning orthogonal polynomials. Trans. Amer. Math. Soc. 37 (1935) 196-206.

[21] R. Teodorescu, E. Bettelheim, O. Agam, A. Zabrodin and P. Wiegmann, Normal random matrix ensemble as a growth problem. Nucl. Phys. B $\mathbf{7 0 4}$ (2005) 407-444.

[22] A. Varchenko and P. Etingof, Why the Boundary of a Round Drop Becomes a Curve of Order Four. American Mathematical Society AMS University Lecture Series. Providence, Rhode Island (1992).

[23] P. Wiegmann and A. Zabrodin, Conformal maps and integrable hierarchies. Comm. Math. Phys. 213 (2000) $523-538$. 